\title{
Formation and evolution of galactic spheroids by mergers
}

\author{
Thorsten Naab $^{1} \dagger$, Andreas Burkert ${ }^{1}$, Peter H. Johansson ${ }^{1}$ and \\ Roland Jesseit ${ }^{1}$ \\ ${ }^{1}$ University Observatory Munich, Scheinerstr. 1, 81679 Munich, Germany \\ email:naab@usm.lmu
}

\begin{abstract}
Galactic spheroids can form as a result of galaxy interactions and mergers of disks. Detailed analyses of the photometric properties, the intrinsic orbital structure, the line-of-sight velocity distributions and the kinemetry of simulated merger remnants, which depend critically on the geometry and the gas content of the interacting progenitors, indicate that low and intermediate mass rotating ellipticals can form from mergers of disks. The masses and metallicities of all massive ellipticals and the kinematics of some massive non-rotating ellipticals cannot be explained by binary mergers. Thus these galaxies might have formed in a different way.
\end{abstract}

Keywords. galaxies: elliptical and lenticular, cD, galaxies: evolution, methods: numerical

\section{The disk merger hypothesis}

Following the first fully self-consistent simulations of mergers of stellar disk galaxies performed by Gerhard (1981) and Negroponte \& White (1983) a large number of increasingly sophisticated simulations have been performed to test whether disk mergers can form elliptical galaxies (see e.g. Barnes \& Hernquist 1992). After more than three decades of research we can summarize the situation as follows: global properties of disk merger remnants are in several respects consistent with observations of giant elliptical galaxies, e.g. equal mass remnants are triaxial and slowly rotating, have boxy or disky isophotes (Heyl et al. 1994, Naab, Hernquist \& Burkert 1999, Naab \& Burkert 2003). In addition, mergers of disks can result in the formation of kinematic subsystems like kinematically decoupled cores at the centers of ellipticals (Hernquist \& Barnes 1991, Jesseit et al. 2007) as well as observed faint structures like shells, loops and ripples at large radii. Unequal mass mergers are more supported by rotation and have disky isophotes (Naab \& Burkert 2003). Collisionless merger remnants are dominated by box orbits at their centers and tube orbits in the outer parts. The total fraction of tube to box orbits increases with the mass ratio of the mergers (Jesseit et al. 2005) and it also correlates with the shape and kinematics of the systems. In addition the mix of disky and boxy isophotal shapes for equal-mass remnants can be understood by the projected properties of tube orbits in triaxial potentials (Jesseit et al. 2005, Naab et al. 2006a). It has been shown by Barnes \& Hernquist (1996) that gas accumulating at the center of merger remnants creates a steep cusp in the central potential well resulting in a more axisymmetric central shape of the remnants. At the same time the fraction of stars on box orbits is significantly reduced and tubes become the dominant orbit family. The most reasonable explanation for this behaviour is that systems with steep cusps in their potential cannot sustain a large population of box orbits. One interesting consequence of the change of orbit populations is that the asymmetry of the LOSVD of the stars changes in a way that is consistent with

$\dagger$ email: naab@usm.lmu.de 


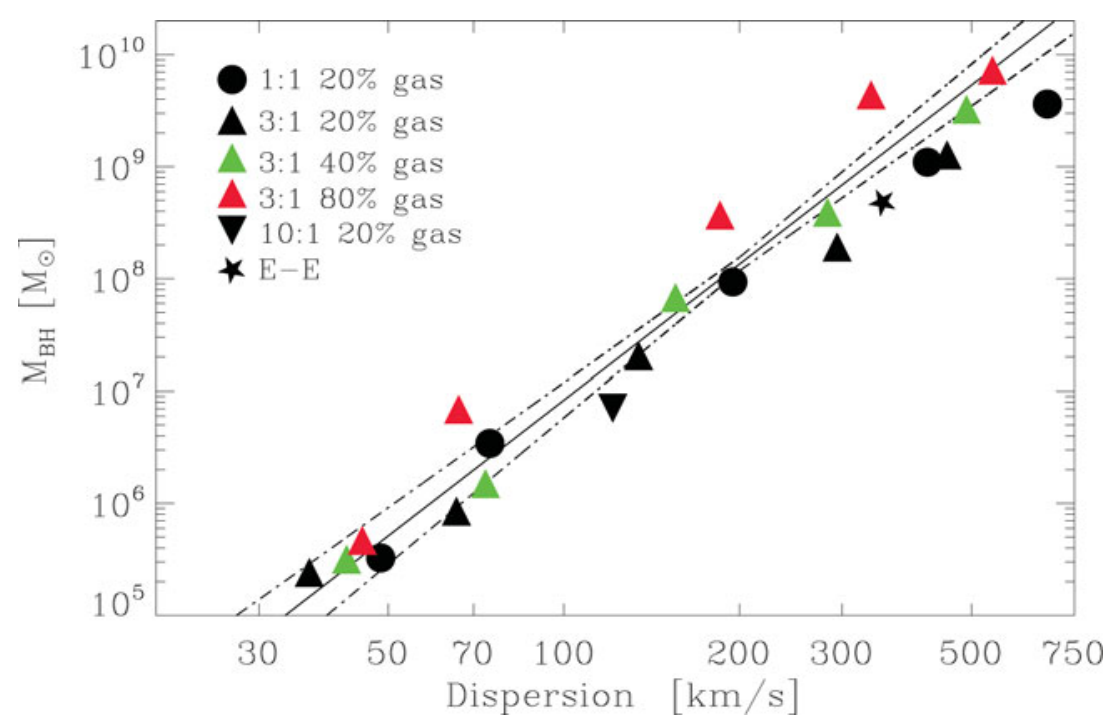

Figure 1. The final stellar velocity dispersion within the effective radius plotted against the final black hole mass for our sample of 1:1, 3:1, 10:1 and E-E mergers. We overplot our simulated data with the Tremaine et al. (2002) fit (solid line) to the observations together with their fitted $1 \sigma$ limits (dashed lines). The simulated merger remnants follow the observed $M_{\mathrm{BH}}-\sigma$ relation with the scatter primarily caused by the initial gas mass fraction.

observations making it a good dynamical tracer for the presence of gas during the merger (Naab et al. 2006a). In addition, this asymmetry can also be thought of as a consequence of two distinct components, e.g. a hot spheroidal bulge and a rotationally supported cold disk as investigated by Naab \& Burkert (2001).

Naab \& Burkert (2003) and Naab \& Trujillo (2006) have argued, based on statistics of kinematic and photometric properties of equal- and unequal-mass mergers, that disk mergers (with bulges) can result in low and intermediate mass elliptical galaxies, as seen in local observed disk mergers (Dasyra et al. 2006a, 2006b, Väisänen et al. 2007). But the objects formed in the simulations are not in agreement with the most massive, boxy and slowly rotating ellipticals which might have assembled by collisionless mergers of earlytype galaxies as indicated by observational as well as theoretical evidence (Khochfar \& Burkert 2003, 2005, Khochfar \& Silk 2006, Naab et al. 2006b, Bell et al. 2006).

Dissipational merging, including star formation, can also overcome stellar phase space constraints and therefore the a priori inclusion of a bulge component is not necessary. It has also been shown by Robertson et al. (2006) that a progenitor gas fraction of 30 per cent results in remnants with parameters in good agreement with the Fundamental Plane for elliptical galaxies. Using a simple model for gas accretion onto a central supermassive black hole it has been argued, based on simulations of binary disk mergers, that gas inflow regulated by black hole feedback can naturally explain the observed present day relation between stellar velocity dispersion and black hole mass for elliptical galaxies and their evolution with redshift (Di Matteo et al. 2005). Using the same approach we simulated a large sample of unequal mass binary mergers including radiative gas cooling, star formation, as well black hole growth and the associated feedback processes (see Johansson et al. 2007 in prep and Springel et al. (2005) for details on the feedback model). We find that unequal 3:1 mergers follow the same $M_{\mathrm{BH}}-\sigma$ relation established for 1:1 mergers, with the scatter primarily caused by the initial gas mass fraction (see Fig. 1). In addition we find evidence that also lower ratio mergers of 10:1, as well as 


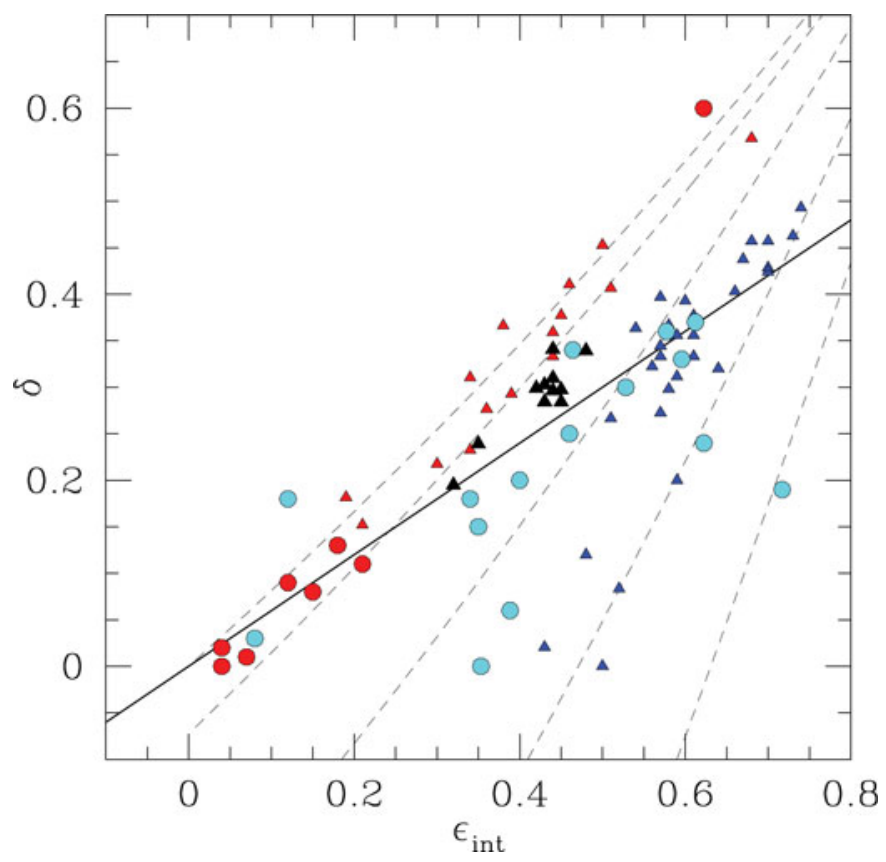

Figure 2. The anisotropy $\delta$ versus the edge-on projected ellipticity $\epsilon_{\text {int }}$ is shown for SAURON early-type galaxies (slow rotators: red circles, fast rotators: cyan circles) and dynamically relaxed merger remnants of disk galaxies mass ratios of 1:1 (red triangles) and 3:1 (blue triangles). Mergers between early-type galaxies are shown as black triangles. The solid line is the correlation derived from modelling given by Cappellari et al. (2007). Dashed lines show from left to right the theoretically predicted correlation $\delta(c)$ for constant values of $v / \sigma=0,0.25,0.5,0.75,1$, respectively. No simulated binary merger covers the region of the slowly rotating SAURON ellipticals, not even early-type mergers.

re-simulations of the merger remnants ('E-E' mergers) follow this same universal correlation between the final black hole mass and the stellar velocity dispersion.

Extended models based on mergers in a cosmological context by Hopkins et al. (2006) have also been used to explain the evolution of Quasars and stellar spheroids as a whole. However, recent studies of dissipative mergers by Naab et al. (2006a), and, including star formation and feedback processes, by Cox et al. (2006) confirm and strengthen the Naab \& Burkert (2003) conclusion that binary disk mergers are reasonable progenitors of intermediate mass giant ellipticals but not for the more massive ellipticals.

\section{The challenges}

Still, serious unsolved problems remain as typical disks are less massive and metal rich than typical ellipticals (Naab \& Ostriker 2006, 2007). It has been shown by Cretton et al. (2001) that the outer kinematics of low mass ellipticals cannot be explained by unequal mass mergers, a problem not yet solved. Additionally, gas-poor as well as gas-rich disk mergers fail to fit the correlation between anisotropy $\delta$ and ellipticity $\epsilon_{\text {int }}$ inferred from Schwarzschild modelling of SAURON ellipticals (Cappellari et al. 2007). In particular, there seems to be no way to produce round, isotropic and slowly rotating systems by binary mergers, not even by mergers of ellipticals (see Fig. 2). Future investigations, including a detailed analysis of simulated merger remnants using orbit modelling techniques (Thomas et al. 2007) will help to understand this discrepancy. It might turn out that 
massive ellipticals cannot be made by binary mergers but form in a more complicated way that can only be investigated using detailed high resolution cosmological simulations as outlined in Naab et al. (2007).

\section{References}

Barnes, J. E. \& Hernquist, L. 1992, ARAA, 30, 705

Barnes, J. E. \& Hernquist, L. 1996, ApJ, 471, 115

Bell, E. F., et al. 2006, ApJ, 640, 241

Cappellari, M., et al. 2007, MNRAS, 379, 418

Cox, T. J., Dutta, S. N., Di Matteo, T., Hernquist, L., Hopkins, P. F., Robertson, B., \& Springel, V. 2006, ApJ, 650, 791

Cretton, N., Naab, T., Rix, H.-W., \& Burkert, A. 2001, ApJ, 554, 291

Di Matteo, T., Springel, V., \& Hernquist, L. 2005, Nature, 433, 604

Dasyra, K. M., et al. 2006a, ApJ, 638, 745

Dasyra, K. M., et al. 2006b, ApJ, 651, 835

Gerhard, O. E. 1981, MNRAS, 197, 179

Hernquist, L. \& Barnes, J. E. 1991, Nature, 354, 210

Heyl, J. S., Hernquist, L., \& Spergel, D. N. 1994, ApJ, 427, 165

Hopkins, P. F., Hernquist, L., Cox, T. J., Di Matteo, T., Robertson, B., \& Springel, V. 2006, ApJS, 163, 1

Jesseit, R., Naab, T., \& Burkert, A. 2005, MNRAS, 360, 1185

Jesseit, R., Naab, T., Peletier, R. F., \& Burkert, A. 2007, MNRAS, 376, 997

Khochfar, S. \& Burkert, A. 2003, ApJ Letters, 597, L117

Khochfar, S. \& Burkert, A. 2005, MNRAS, 359, 1379

Khochfar, S. \& Silk, J. 2006, MNRAS, 370, 902

Naab, T., Burkert, A., \& Hernquist, L. 1999, ApJ (Letters), 523, L133

Naab, T. \& Burkert, A. 2001, ApJ (Letters), 555, L91

Naab, T. \& Burkert, A. 2003, ApJ, 597, 893

Naab, T. \& Ostriker, J. P. 2006, MNRAS, 366, 899

Naab, T. \& Trujillo, I. 2006, MNRAS, 369, 625

Naab, T., Jesseit, R., \& Burkert, A. 2006a, MNRAS, 372, 839

Naab, T., Khochfar, S., \& Burkert, A. 2006b, ApJ Letters, 636, L81

Naab, T., Johansson, P. H., Ostriker, J. P., \& Efstathiou, G. 2007, ApJ, 658, 710

Naab, T. \& Ostriker, J. P. 2007, ArXiv Astrophysics e-prints, arXiv:astro-ph/0702535

Robertson, B., Cox, T. J., Hernquist, L., Franx, M., Hopkins, P. F., Martini, P., \& Springel, V. 2006, ApJ, 641, 21

Springel, V., Di Matteo, T., \& Hernquist, L. 2005, MNRAS, 361, 776

Tremaine, S. et al., 2002, ApJ, 574, 740

Thomas, J., Jesseit, R., Naab, T., Saglia, R. P., Burkert, A., \& Bender, R. 2007, ArXiv e-prints, 708, arXiv:0708.2205

Väisänen, P., et al. 2007, ArXiv e-prints, 708, arXiv:0708.2365 